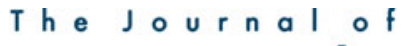 \\ Laryngology \& Otology \\ ENT trainee papers presented at the ENT Scotland Winter Meeting, 18 November 2016, Sterling, Scotland, UK
}

cambridge.org/jlo

\section{Online Only Abstract Selection}

Cite this article: ENT trainee papers presented at the ENT Scotland Winter Meeting, 18 November 2016, Sterling, Scotland, UK. J Laryngol Otol 2018;E5:1-4. https://doi.org/ $10.1017 /$ S0022215118000403

First published online: 8 June 2018
Primary versus secondary tracheoesophageal puncture: a systematic review and meta-analysis

P D Chakravarty, A E L McMurran, A Banigo, M Shakeel and K W Ah-See

From Aberdeen Royal Infirmary

\section{Introduction}

Tracheoesophageal puncture represents the 'gold standard' for voice restoration following laryngectomy. Tracheoesophageal puncture can be undertaken primarily during laryngectomy or in a separate secondary procedure. There is no current consensus on which approach is superior.

Aim

To assess current evidence comparing primary and secondary tracheoesophageal puncture.

\section{Methods}

A systematic review and meta-analysis of primary versus secondary tracheoesophageal puncture were conducted. Outcomes assessed were: voice success, overall complication rate and pharyngocutaneous fistula rate.

Results

Twelve case series, 10 retrospective and 2 prospective studies, met the inclusion criteria. The meta-analysis results did not demonstrate a statistically significant difference in overall complication rate, though a secondary tracheoesophageal puncture approach was favoured to avoid pharyngocutaneous fistula. The evidence trends towards primary tracheoesophageal puncture for voice success, though this did not reach statistical significance.

\section{Conclusions}

There is no statistically significant difference in outcomes when primary and secondary tracheoesophageal puncture approaches are compared, but if there is an increased risk of pharyngocutaneous fistula a secondary approach should be favoured.

Post-tonsillectomy outcomes versus parental pre-operative expectations. How did we do?

T Whittle and S Benzie

From Crosshouse University Hospital, Kilmarnock

Problem

Extensive research has shown the benefit of tonsillectomy in children. Few studies have assessed parental expectation before surgery in comparison to post-operative outcomes.

Aim

To ascertain how parental expectations pre-tonsillectomy compare to parent-rated outcomes following surgery.

Methods

A questionnaire rating eight possible benefits of tonsillectomy was completed by parents prior to surgery and at least six months after the operation along with a Children's Glasgow Benefit Inventory. Pre-operative expectations were compared with parent-rated post-operative results and the Children's Glasgow Benefit Inventory.

Results

Fifty-eight per cent of parents $(n=40)$ returned the post-operative questionnaire. The global Children's Glasgow Benefit Inventory mean score was +49 . The procedure was least beneficial for children with sleep-disordered breathing (Children's Glasgow Benefit 
Inventory score of +41 ). Physical outcomes exceeded expectations, most markedly in the recurrent tonsillitis cohort. Social outcomes failed to meet expectations, most notably in the sleep-disordered breathing cohort.

\section{Conclusion}

While the procedure was felt to be of benefit, parents underestimated the physical benefits of tonsillectomy and overvalued the social benefits of the procedure. Better understanding of parental expectations can potentially improve pre-operative counselling.

\section{Different pathways in head and neck cancers - a need} for change

R Crosbie, A Scott, J McMahon and J Montgomery

From the Queen Elizabeth University Hospital, Glasgow

\section{Introduction}

A diagnosis of head and neck cancer is exceptionally distressing for patients and families. Optimising intervals between referral and treatment is imperative, and is a 'HEAT' performance target of the Scottish government.

\section{Methods}

All new cancer cases discussed by the South Glasgow multidisciplinary team between June 2014 and January 2016 were included. A case note review was performed, and intervals between referral, out-patient clinic, cross-sectional imaging and treatment were recorded. Differences between specialties were explored.

\section{Results}

A total of 312 new cancer cases were included: 170 ENT, and 142 oral and maxillofacial surgery cancers. Regarding these two groups, mean patient age was 63.1 and 64.8 years respectively ( $p=0.2135)$, time between referral and out-patient clinic attendance was 22.8 versus 11.3 days $(p<0.0001)$, time between out-patient clinic attendance and axial imaging was 21.8 versus 7.3 days $(p<0.0001)$, and time between referral and treatment was 85.1 versus 57.7 days $(p<0.0001)$. The pathway of patients with suspicion of cancer was also compared, with similar statistically different results.

\section{Conclusions}

There is a gross inequality between patients with cancers in neighbouring head and neck sites. This must be addressed and optimised.

Does the use of an internal auditory meatus protocol in brain magnetic resonance imaging detect incidental findings that require attention?

W B Cheang ${ }^{1}$, T Sudarshan ${ }^{1,2}$ and M Hussain ${ }^{1,2}$

From the ${ }^{1}$ University of Dundee School of Medicine and ${ }^{2}$ Ninewells Hospital, Dundee

\section{Introduction}

A significant proportion of imaging studies reveal unexpected, incidental radiological findings.
Aim

To evaluate the frequency of incidental findings that require further attention on magnetic resonance imaging (MRI) scans of patients with audiovestibular symptoms.

\section{Method}

A retrospective study was conducted of 2049 MRI scans.

Results

Gender distribution was equal. Patients' age ranged from 7 to 91 years (mean, 56 years). Of the scans, 801 (39 per cent) were normal and 21 (1 per cent) demonstrated a unilateral acoustic neuroma. A total of 1227 scans (59.9 per cent) demonstrated incidental findings. Of the scans, 1136 (55.4 per cent) were considered to be of no or little clinical significance; these revealed mainly age-related changes (30.1 per cent), mucosal inflammation (16 per cent) and vascular anomalies (9.7 per cent). A total of 104 scans (5 per cent) were considered clinically significant and warranted appropriate referral; these mainly revealed extensive white matter lesions ( 2.4 per cent), meningiomas ( 0.5 per cent) and aneurysms ( 0.5 per cent).

\section{Conclusion}

The use of an internal auditory meatus protocol in brain MRI successfully detects incidental findings that require attention. These were observed in 5 per cent of scans.

Is antibiotic prophylaxis required in mastoid surgery? A comparative case series study and survey of national practice

\section{Graham, K McAllister, A Clement and S Morrissey}

From the Royal Hospital for Sick Children, Glasgow

Aims

This prospective pilot study aimed to assess the impact of prophylactic antibiotic use in mastoid surgery. The study also aimed to examine Scottish otologists' usage of antibiotics via a national survey.

\section{Methods}

A retrospective case note review was conducted of all elective mastoid operations performed at Glasgow's Royal Hospital for Sick Children from June 2013 to August 2016. The primary outcomes examined were wound infection and otorrhoea, post-operatively. A survey of Scottish otologists was also conducted, examining prophylactic antibiotic prescription in mastoid surgery.

\section{Results}

Seventy-four operations were reviewed. Prophylactic antibiotics were used in 29 cases and no prophylaxis was administered in 45 . Of the 29 antibiotic group cases, only 1 wound infection resulted ( 3.4 per cent), compared to 3 wound infections in the 45 non-antibiotic group cases (6.7 per cent). Otorrhoea was compared between the antibiotic and nonantibiotic groups post-operatively at the following time points: within the first month (31.0 per cent vs 26.75 per cent), between the first and third month (30.4 per cent $v s 42.2$ per cent), and between the third and sixth month (28.6 per cent vs 28.9 per cent). The survey response rate was 58.3 per cent. The results showed that 35.7 per cent of the otologists who responded to the survey regularly use prophylactic 
antibiotics in mastoid surgery, for infective mucosal disease and infected cholesteatoma.

\section{Conclusion}

Antibiotic prophylaxis does not appear to affect post-operative otorrhoea rates in patients undergoing mastoid surgery. Post-operative wound infections are uncommon following these operations. Our survey of otologists demonstrated a significant variation in antibiotic prescription when operating on infected or discharging ears. This is a potential area to direct further research.

\section{Epistaxis management - the East of Scotland experience}

S Kaushal, L Nelson, R Green and P Spielmann

From the Ninewells Hospital, Dundee

\section{Background}

Epistaxis is one of the commonest ENT emergencies. Most hospitals do not have standardised protocols for epistaxis treatment, which increases the potential for prolonged admissions and re-admissions.

\section{Methods and results}

A retrospective review was conducted of the medical notes of epistaxis patients admitted to four ENT units (Dundee, Edinburgh, Aberdeen and Inverness) between August and November 2015, and several outcomes were assessed and compared. Despite similar patient demographics, there were considerable differences in management between units, both in accident and emergency (A\&E) departments and on the ENT wards. This may account for the variations in length of stay and re-admission rates.

\section{Conclusion}

Our audit has highlighted areas where education of A\&E and ENT staff would be beneficial in optimising the care of epistaxis patients. There is also the need for an anticoagulation/ antiplatelet therapy protocol in epistaxis cases. We plan to re-audit performance across all units as part of the Integrate British audit.

Post-operative grommet review at a parallel audiology clinic: a cost-effective approach to reduce pressure at ENT clinics?

N Jacob ${ }^{1}$, C Doherty $^{1}$, L Hamilton ${ }^{2}$ and D Wynne ${ }^{2}$

From the ${ }^{1}$ University of Glasgow and ${ }^{2}$ Royal Hospital for Children, Glasgow

\section{Introduction}

There is significant pressure on ENT clinics to see new patients on their waiting list, in addition to reviewing patients. At the Royal Hospital for Children, approximately 350 grommet operations are conducted annually. These patients require review to check the patency of grommets and hearing. Traditionally, this was done at the ENT clinic with additional input from audiology. It is proposed that the audiology department could be trained to run a parallel clinic to review these patients without ENT departmental input, to reduce the clinical workload of the ENT department.
Aim

To determine if an audiology department led clinic is a costeffective alternative to the ENT clinic for post-grommet review.

\section{Method}

A departmental audit was conducted, with a retrospective review of all children who underwent grommet insertion at the Royal Hospital for Children between February 2014 and August 2016.

Results

A total of 128 children were included in the study. Sixty-four (48 per cent) were seen and discharged by the audiology department alone and within four months of surgery.

\section{Conclusion}

Our study supports the introduction of an audiology department led parallel clinic to review post-operative grommet patients, to increase availability and reduce costs of the ENT clinic.

Assessment of vestibular function in patients with superior semicircular canal dehiscence: pilot study of the role of the six-canal video head impulse test

\section{S J Messeder ${ }^{1,2}$, M Gaggini ${ }^{2}$ and G Kontorinis ${ }^{2}$}

From the ${ }^{1}$ University of Glasgow and ${ }^{2}$ Queen Elizabeth University Hospital, Glasgow

\section{Introduction}

Superior semicircular canal dehiscence is a rare condition in which individuals experience vertigo, often triggered by loud noises, or changes in middle-ear pressure and autophony due to the third window effect.

\section{Aims}

We examined the role of the six-canal video head impulse test in assessing and monitoring patients with superior semicircular canal dehiscence.

\section{Methods}

A prospective pilot study is being conducted in a tertiary, referral centre. Currently, eight patients diagnosed with superior semicircular canal dehiscence have undergone pure tone audiometry, tympanometry, dynamic posturography, cervical vestibular-evoked myogenic potentials testing and a video head impulse test.

\section{Results}

All patients presented with typical superior semicircular canal dehiscence symptoms: mild low-frequency conductive hearing loss and increased amplitude on vestibular-evoked myogenic potentials testing. The dehiscence was confirmed by temporal bone computed tomography. Patients had abnormalities in their video head impulse test findings, but not always of the superior canal.

\section{Conclusion}

Our preliminary results indicate that the video head impulse test is a simple additional test for patients with superior semicircular canal dehiscence. Further experience will explain the 
underlying mechanism, and might facilitate post-operative confirmation of successful canal obliteration or reconstruction.

\section{Breaking 'NEWS': scoring systems in ENT emergency admissions}

J Morton, R Hurley and S Keh

From the Queen Elizabeth University Hospital, Glasgow, UK

\section{Introduction}

The National Early Warning Score ('NEWS'), systemic inflammatory response syndrome ('SIRS') and quick sepsis-related organ dysfunction assessment ('qSOFA') are used to identify deteriorating patients.

Aim

This study examined the use of these scoring systems in an ENT environment.

\section{Method}

A 100-day retrospective review was conducted of adult emergency admissions under ENT in NHS Greater Glasgow and Clyde. Descriptive statistics were used for analysis.

\section{Results}

A total of 101 emergency admissions were examined. The mean National Early Warning Score on admission was 1.18. There was significant improvement in the National Early Warning Score upon discharge. Twenty-nine per cent had systemic inflammatory response syndrome with evidence of infection. No patients scored 2 or more on the quick sepsisrelated organ dysfunction assessment. Ten per cent of patients' National Early Warning Scores deteriorated during admission.

\section{Conclusions}

The National Early Warning Score at admission reflects the wellness of ENT patients at presentation, and improvement is consistent with suitable intervention. A high National Early Warning Score was used to escalate deteriorating patients appropriately. Patients were not documented as septic, and the 'sepsis six' bundle of therapies was never implemented - this did not lead to negative outcomes. It is unclear if low National Early Warning Scores provided reassurance, or if the quick sepsisrelated organ dysfunction assessment is a more specific indicator of sepsis in ENT patients.

\section{Management of nasal septal perforation using a Silastic button: Grampian experience}

\section{Edmond and M Shakeel}

From Aberdeen Royal Infirmary

\section{Introduction}

Many patients with nasal septal perforation seek our help with symptoms such as crusting, whistling and epistaxis. Surgical repair has a low success rate. Blocking the nasal septal perforation with an obturator is an alternative option.

Aims

To assess the effectiveness of, and patients' compliance with, the Silastic button for nasal septal perforation.

\section{Methods}

A retrospective analysis was conducted of the case records for all patients who have undergone septal button insertion at Aberdeen Royal Infirmary since 2000. Patients were identified from operating theatre logbooks. Data collected included demographics, presenting symptoms, post-operative symptoms and complications.

Results

A total of 70 patients were identified: 44 females and 26 males. Symptom improvement was reported in 66 per cent of patients $(n=46)$. Thirty-four per cent of patients $(n=24)$ had worsening of symptoms, or required button replacement, trimming or removal.

\section{Conclusions}

The Silastic button is an alternative treatment modality for nasal septal perforation. However, it still carries high complication and failure rates. 